Influence of interparticle interaction on melting of gold nanoparticles in Au/ polytetrafluoroethylene nanocomposites

Oleg A. Yeshchenko', Igor M. Dmitruk, Konstantin P. Grytsenko, Vadym M. Prokopets, Andriy V. Kotko, and Sigurd Schrader

Citation: Journal of Applied Physics 105, 094326 (2009); doi: 10.1063/1.3125274

View online: $\mathrm{http}: / / \mathrm{dx}$.doi.org/10.1063/1.3125274

View Table of Contents: http://aip.scitation.org/toc/jap/105/9

Published by the American Institute of Physics

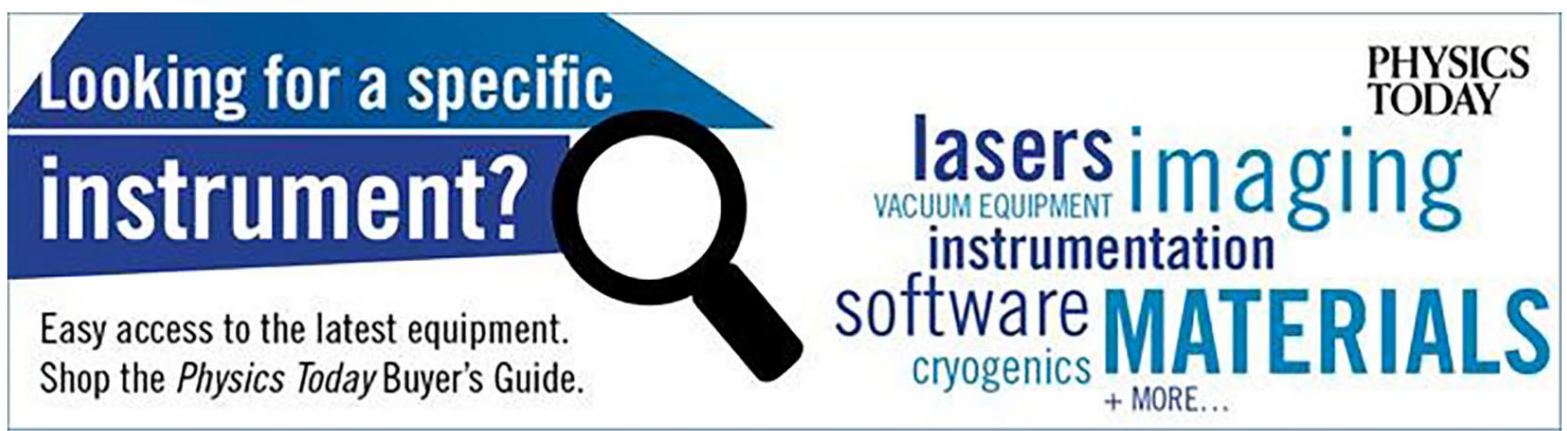




\title{
Influence of interparticle interaction on melting of gold nanoparticles in Au/polytetrafluoroethylene nanocomposites
}

\author{
Oleg A. Yeshchenko, ${ }^{1, a)}$ Igor M. Dmitruk, ${ }^{1}$ Konstantin P. Grytsenko, ${ }^{2}$ Vadym M. Prokopets, ${ }^{1}$ \\ Andriy V. Kotko, ${ }^{3}$ and Sigurd Schrader ${ }^{4}$ \\ ${ }^{1}$ Department of Physics, National Taras Shevchenko Kyiv University, 2/1 Akademik Glushkov Prosp., \\ 03127 Kyiv, Ukraine \\ ${ }^{2}$ V. E. Lashkaryov Institute of Semiconductor Physics, 45 Nauky Prosp., 03650 Kyiv, Ukraine \\ ${ }^{3}$ I. M. Frantsevich Institute for Problems of Materials Science, 3 Krzhizhanovsky Str., 03680 Kyiv, Ukraine \\ ${ }^{4}$ Institute of Photonics, Laser \& Plasma Technology, University of Applied Sciences, 63 F.-Engels-Str., \\ 15745 Wildau, Germany
}

(Received 2 October 2008; accepted 29 March 2009; published online 12 May 2009)

\begin{abstract}
The temperature dependence of the surface plasmon resonance energy and width in $5 \mathrm{~nm}$ spherical gold nanoparticles embedded in a polymer polytetrafluoroethylene matrix has been studied using absorption spectroscopy. The jumplike features have been observed in these dependences indicating the melting of gold nanoparticles at temperatures considerably lower than the bulk melting point. The interaction between gold nanoparticles sufficiently affects the melting of nanoparticles. The increase in the filling factor of the particles leads to a decrease in the melting temperature of gold nanoparticles. (C) 2009 American Institute of Physics. [DOI: 10.1063/1.3125274]
\end{abstract}

\section{INTRODUCTION}

Crystalline materials loose their crystal order and become a liquid during melting transformation. Melting of small nanometer-sized particles differs from that of bulk materials (see, e.g., Refs. 1-4). When the size of a particle decreases, its surface to volume ratio increases inversely proportional to the radius of the particle. As a result, the surface atoms become loosely bounded and diffuse on the surface. Surface diffusion is followed by a partial melting of the nanoparticle mainly within a thin layer close to the surface, which is called surface melting or premelting. ${ }^{5-7}$ The surface melting generally occurs at temperatures much lower than the bulk melting but the details depend on the parameters such as size, ${ }^{1}$ morphology ${ }^{8}$ defects, ${ }^{9}$ strain, ${ }^{3}$ and crystallographic orientation of the surface. ${ }^{1,3}$ The interior or volume melting of a nanoparticle takes place at a melting temperature $\left(T_{m}\right)$ lower than the bulk melting point $\left(T_{\infty}\right)$. The decrease in $T_{m}$ with the decrease in the particle size is a well known phenomenon that has been described in terms of phenomenological thermodynamic models, ${ }^{10}$ and, more recently, simulated using molecular dynamics. ${ }^{5-7,11-13}$ Experimental evidence of the decrease in $T_{m}$ has been demonstrated by a variety of techniques. ${ }^{2,14-18}$ In addition, the influence of surrounding matrix on $T_{m}$ has been demonstrated, e.g., in our previous work. ${ }^{19}$ However, the influence of the interaction between the metal nanoparticles (or filling factor of nanocomposite) on their melting point is an unstudied problem.

The optical properties of nanocomposite consisting of metal nanoparticles embedded in a dielectric matrix are mainly determined by the surface collective oscillations of free (conduction) electrons or surface plasmons (SPs). The energy and damping constant of SP resonance depend on dielectric functions of nanoparticle and surrounding matrix.

${ }^{a)}$ Electronic mail: yes@univ.kiev.ua.
The SP energy and damping constant are very sensitive to the changes in structure, aggregation state of nanoparticle (solid, liquid, or vapor), its size, shape, etc., as well as external influences caused, e.g., by matrix or interaction with other nanoparticles. Therefore, the spectral characteristics of SP absorption band (the spectral position and bandwidth) are a very appropriate tool to reveal these changes. Optical properties of gold nanoparticles are quite remarkable because the energy of the SPs mostly coincides with the onset of interband transitions. Thus, the optical spectroscopy (absorption in part) gives an opportunity to study the influence of both valence and conduction electrons on optical properties of gold nanoparticles.

In this paper we report the influence of the interaction between gold nanoparticles embedded in $\mathrm{Au} /$ polytetrafluoroethylene (PTFE) nanocomposites on melting point of spherical gold nanoparticles using the absorption spectroscopy, which, to our best knowledge, has not been reported so far. As we already noted, the melting point of metals decreases when the size of the nanoparticles decreases. Since metal nanoparticles have been widely used in electronic and optoelectronic industry, the determination of the maximum allowed temperature for their exploitation is a very important technological problem. This information is obtained from the experiments of metal nanoparticle melting. Moreover, the optical spectroscopy is a nondestructive tool for determination of the extremal characteristics of exploitation regimes of nanometal composites and electronic devices built using these composite nanomaterials. On the other hand, with the development of nanotechnology, the sufficient utilization of the low melting point property of nanosized materials is paid more and more attention. Many experimental results showed that the melting point of nanoparticles is low, but the melting point increases after melting and merging of small nanoparticles resulting in the formation of large particle with high melting point close to $T_{\infty}$. This character- 
istic just satisfies the demands for low temperature in manufacturing technique and high temperature in outer environment in the microelectronics devices, which may resolve the problems of materials for solder joints. Besides the technological interest, the melting point is of fundamental scientific interest. In most reports the melting of nanoparticles has been detected by high resolution transmission electron microscopy (HRTEM) and electron diffraction techniques. However, both techniques use the samples that are sufficiently heated by the electron beam. Therefore, there is no possibility to control the temperature of the sample. On the other hand, the absorption spectroscopy gives such a possibility, i.e., the temperature determined in our work is the real temperature of the sample. The study of the temperature dependences of the SP energy and bandwidth in gold nanoparticles at various values of filling factor allowed us to reveal the effects of influence of interaction between the gold nanoparticles on their melting characteristics.

\section{FABRICATION OF AU/PTFE NANOCOMPOSITES: EXPERIMENTAL PROCEDURES}

The samples were fabricated by gold and polymer PTFE coevaporation in a vacuum. PTFE pellets were evaporated with vapor activation by electron cloud. Additional activation of both vapors and substrate surface was done by $\mathrm{rf}$ discharge. The discharge frequency of $40.68 \mathrm{MHz}$ was chosen as one of available industrial ones suitable for matching with the PTFE vacuum deposition conditions. The higher the frequency and electron concentration, the lower the energy of the electrons, which creates favorable conditions for polymerization. This method was described in details in Refs. 20 and 21 . Au was evaporated using tantalum boat. The films were deposited using UVN-74P3 installation equipped with computerized control system. Starting pressure was $10^{-3} \mathrm{~Pa}$. Rotating glass disk was used as a substrate. Several different shields for $\mathrm{Au}$ vapors were used, so during one deposition films with different Au concentrations (different filling factors) were obtained on the substrate at the same conditions. StellarNet spectrometer with optical fibers was used to record the absorption spectra of the films. Using this procedure, three samples labeled hereafter as AT2, AT3, and AT4 were fabricated. The Au/PTFE film thickness in these samples was measured to be $309 \mathrm{~nm}$ for AT2, $117 \mathrm{~nm}$ for AT3, and $273 \mathrm{~nm}$ for AT4. The filling factors for Au in PTFE matrix in studied samples are the following: $f=0.07$ (AT4), 0.20 (AT2), and 0.34 (AT3).

A tungsten-halogen incandescent lamp was used as a light source for the absorption measurements. The single spectrometer MDR-3 was used for in situ measurements of absorption spectra. Absorption spectra of Au/PTFE nanocomposites were measured in the temperature region of 77 $936 \mathrm{~K}$. The measurements at $77 \mathrm{~K}$ were performed for the samples dipped in the liquid nitrogen and measurements at the room and higher temperatures were performed in air. The samples were placed into an open furnace during the absorption measurements at high temperatures. At the temperature measurements the heating speed was $4 \mathrm{~K} / \mathrm{min}$, and the cooling speed was from $10 \mathrm{~K} / \mathrm{min}$ at sample temperature of 900 $\mathrm{K}$ to $1 \mathrm{~K} / \mathrm{min}$ at $330 \mathrm{~K}$. Transmission electron microscopy

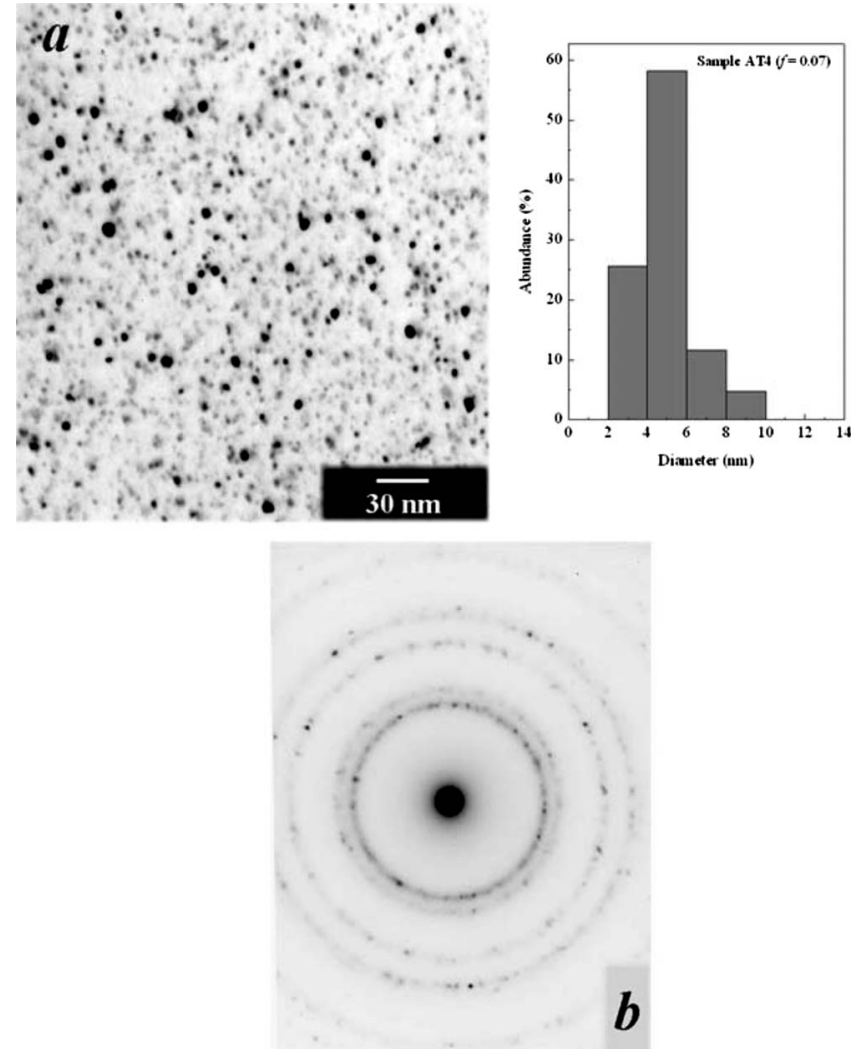

FIG. 1. (a) TEM image and corresponding size distribution of gold nanoparticles in sample AT4 with filling factor of 0.07. (b) Electron diffraction image of gold nanoparticles in sample AT2 with $f=0.20$.

(TEM) and electron diffraction measurements of studied samples were performed by JEOL 100CX electron microscope at room temperature. Atomic force microscopy (AFM) of Au/PTFE samples was performed by Ntegra Solver P47PRO scanning probe microscope at room temperature.

\section{TEM AND ELECTRON DIFFRACTION CHARACTERIZATION OF AU/PTFE NANOCOMPOSITES}

We used a TEM and electron diffraction of fabricated $\mathrm{Au} / \mathrm{PTFE}$ nanocomposites to determine the size and shape distribution of gold nanoparticles in PTFE matrix. The TEM image of sample AT4 with lower filling factor (0.07) is shown in Fig. 1(a). The image proves the creation of the nearly spherical Au nanoparticles with diameters of $5.0 \mathrm{~nm}$ with standard deviation $\sigma=1.5 \mathrm{~nm}$. The TEM images of sample AT4 with lowest filling factor show separate nanoparticles. The TEM images of other samples with higher filling factors prove the formation of nearly spherical gold nanoparticles similar to the ones in sample AT4. However, the reliable statistical analysis of size distribution of nanoparticles is complicated in these samples since the images of most of nanoparticles cannot be separated. Therefore, the electron diffraction study was performed to determine the mean size of gold nanoparticles in samples with high filling factors. The electron diffraction image of sample AT 2 with $f=0.20$ is shown in Fig. 1(b). The comparison of obtained diffraction images with standard gold diffraction patterns proves the for- 


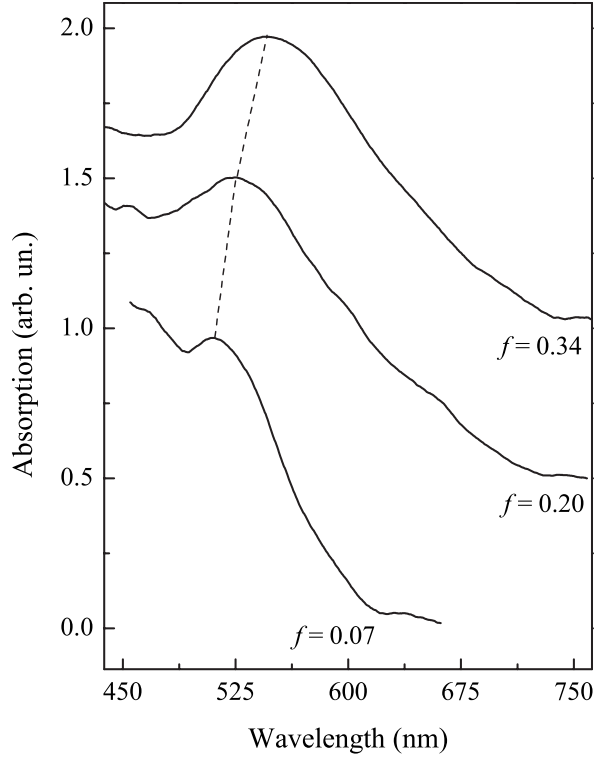

FIG. 2. Absorption spectra of studied Au/PTFE nanocomposites with various filling factor $f$.

mation of gold nanoparticles in PTFE matrix. To determine the mean size $D$ of gold nanoparticles we used the DebyeScherrer expression ${ }^{22,23}$

$$
D=\frac{4}{3} \frac{0.9 \lambda}{\Delta(2 \theta) \cos (\theta)}=\frac{4}{3} \frac{0.9 \lambda L}{\Delta d \cos ^{2}(2 \theta) \cos (\theta)},
$$

where $\Delta(2 \theta)$ is the angular size (half width) of diffraction reflex, $\Delta d$ is the linear size of reflex in registration plane, $\lambda$ is the wavelength of electron beam, $L$ is the distance from sample to the plane of registration (for electron microscope used $\lambda L=3.23 \mathrm{~nm} \times \mathrm{mm}$ ), and $\theta$ is the diffraction angle. As a result, we obtained the diameters of gold nanoparticles in samples AT2 and AT3 to be 5.0 and $5.2 \mathrm{~nm}$. That is in perfect agreement with TEM data. Thus, the size of gold nanoparticles $(5 \mathrm{~nm})$ is the same in all studied samples.

\section{TEMPERATURE DEPENDENCES OF ABSORPTION OF AU/PTFE NANOCOMPOSITES: RESULTS AND DISCUSSION}

The absorption spectra of Au/PTFE nanocomposites prove the existence of gold nanoparticles in our samples (Fig. 2). The spectra demonstrate a clear SP band. With increase in filling factor the plasmon band shifts to low-energy side and broadens. Such behavior of SP band with increase in filling factor is a well known phenomenon. ${ }^{24}$ Gold nanoparticles have the same size in all studied samples. Thus, the observed effects discussed below are not affected by the size and are only filling factor-dependent ones, i.e., these effects are caused by interaction between gold nanoparticles. In nanocomposites with high filling factor (higher than 0.2 ) it is reasonable to expect the aggregation of nanoparticles. However, since any new low-energy bands indicating aggregation are absent in absorption spectra of all three thermally untreated samples, one can conclude that sufficient aggregation

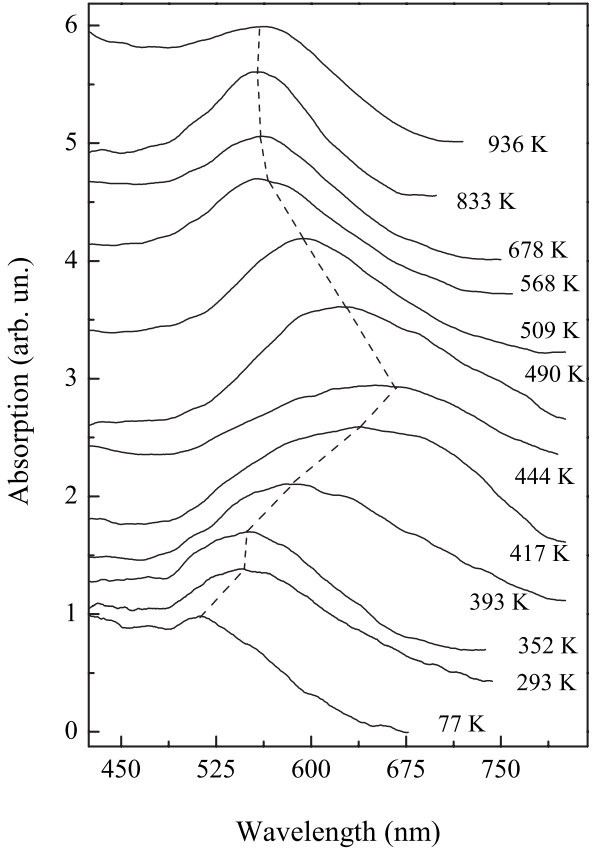

FIG. 3. Evolution of absorption spectra of Au/PTFE nanocomposites with filling factor $f=0.34$ at monotonic increase in temperature.

does not occur in our samples, i.e., the initial optical properties of untreated samples are determined mainly by separated gold nanoparticles.

In the present work we study the temperature dependences of the absorption spectra of Au/PTFE nanocomposites, namely, the temperature dependences of the spectral position of SP absorption band maximum that corresponds to the SP resonance energy and the SP bandwidth. We obtained the values of SP energy and bandwidth from the decomposition of the measured spectrum to basic Lorentzian spectral contours. The evolution of the absorption spectrum of sample AT3 with $f=0.34$ with the monotonically increasing temperature is shown in Fig. 3. One can see a considerable nonmonotonic variation in the SP energy and bandwidth with increase in the temperature. The dependences of SP energy and bandwidth on temperature at monotonically increasing temperature are shown in Fig. 4. The observed changes in spectral characteristics of SP (energy and bandwidth) can be caused by some transformation in the structure of nanoparticles or by transformations in polymer matrix (thermal expansion and softening of polymer matrix). Below, we consider these phenomena as possible causes of observed effects.

Let us note the unexpected nonmonotonic dependence of SP bandwidth at temperatures smaller than the one marked as $T_{p}$ (Fig. 4). At temperatures lower than the room ones (about $293 \mathrm{~K}$ ) the broadening of SP absorption band with increase in temperature is observed, which is due to increase in frequency of electron-phonon scattering. At temperatures higher than the room ones the narrowing of SP absorption band is observed. This is quite an unexpected temperature effect. We assume that this effect is due to two mechanisms. First is the thermal expansion of the PTFE matrix. At the expansion of matrix the distance between the nanoparticles increases which leads to the decrease in the filling factor. It is well 

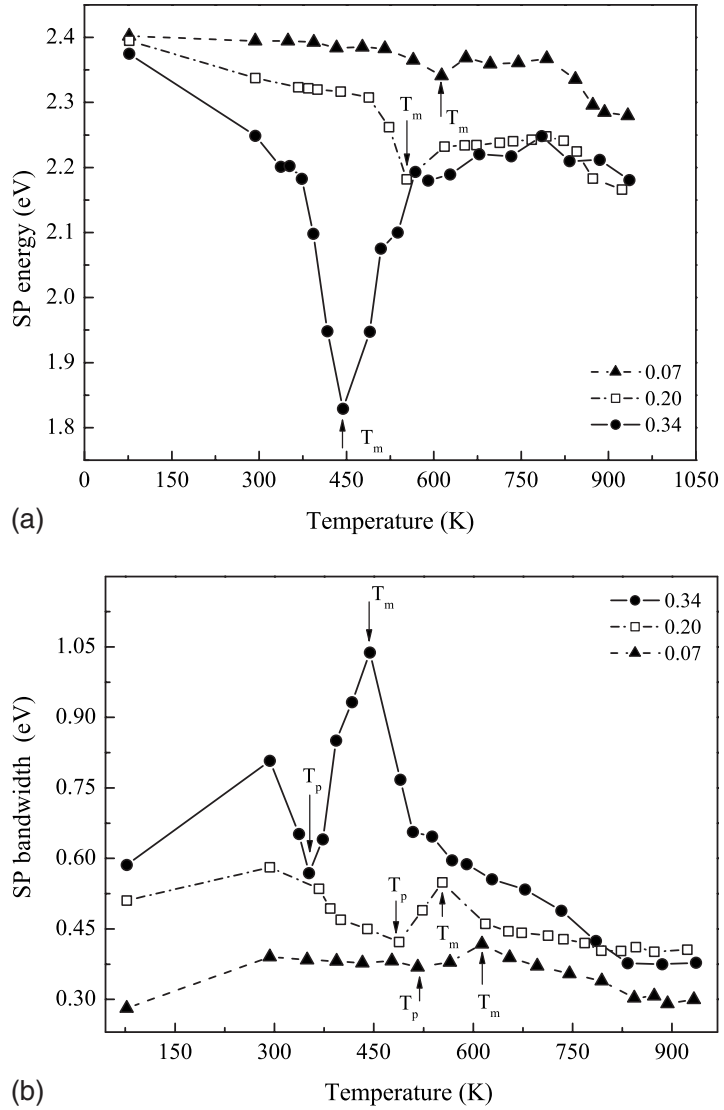

FIG. 4. Temperature dependences of (a) SP energy and (b) bandwidth of gold nanoparticles in PTFE matrix for various filling factors. Absorption measurements were performed at monotonically increasing temperature. $T_{m}$ marks the melting point of Au nanoparticles, $T_{p}$ marks the softening point of PTFE matrix.

known $^{24}$ that at the increase in $f$ the broadening of SP absorption band occurs. That is due to fluctuations of $f$ in the volume of sample. So, at the decrease in the filling factor the narrowing of SP band would occur. Second is the thermal expansion of nanoparticles. It is well known ${ }^{24}$ that SP bandwidth is proportional to the damping constant of the surface plasma oscillations in nanoparticle $\Gamma$, which depends on nanoparticle radius $r$ as

$$
\Gamma=\Gamma_{\infty}+A v_{F} / r,
$$

where $A$ is the proportionality constant, $\Gamma_{\infty}$ is the damping constant for bulk metal, and $v_{F}$ is the Fermi velocity. Therefore, at nanoparticle expansion the SP bandwidth has to decrease. To prove this assumption we studied the reversibility of the temperature dependence of SP energy and bandwidth in the temperature range $293 \mathrm{~K}-T_{p}$ by measuring this dependence, first increasing the temperature from $293 \mathrm{~K}$ to $T_{p}$ and then decreasing $T$ from $T_{p}$ to $293 \mathrm{~K}$. The obtained dependence is shown in Fig. 5. Another explanation of the strong abnormal temperature dependence of SP bandwidth can be either some structural transformations and phase transitions in $\mathrm{Au}$ nanoparticles or nanoparticle aggregation. However phase transitions and aggregation have to lead to hysteresis character of the dependence at the change in heating to cooling and, correspondingly, to irreversibility of the observed dependence. However, we can see from Fig. 5 that
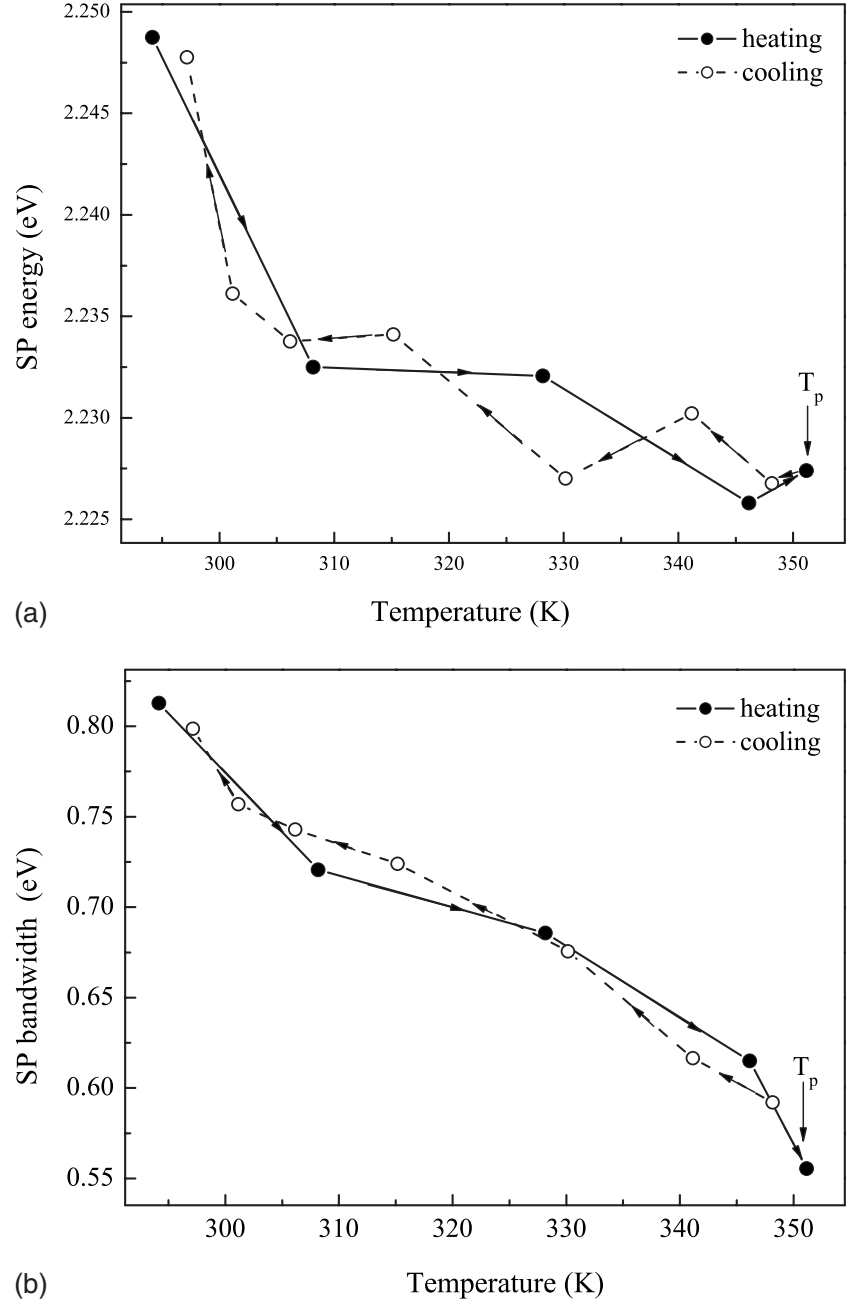

FIG. 5. Reversibility of temperature dependence of (a) SP energy and (b) bandwidth of gold nanoparticles in sample AT3 with $f=0.34$ at small temperature variation under the softening point of PTFE matrix $T_{p}$.

the observed dependence is fully reversible. That proves our assumption of thermal expansion as a cause of decrease in SP bandwidth at the increase in temperature.

At the temperature $T_{p}$ the narrowing of SP absorption band at the increase in temperature changes to its strong broadening. The broadening is followed by strong redshift of SP band (Fig. 4). Most probably, the observed effects are the result of softening of PTFE matrix. The softening makes possible the diffusion of the gold nanoparticles in matrix and their aggregation. The aggregation leads to redshift of SP absorption band and its broadening due to increase in the interaction between the nanoparticles in aggregate. ${ }^{24}$ The aggregation would cause the irreversibility of temperature dependences of SP spectral characteristics at temperatures higher than temperature at which the softening of the matrix starts, i.e., polymer matrix softening point $T_{p}$. To prove this assumption, we measured the temperature dependences of SP energy and bandwidth by first heating the samples from $293 \mathrm{~K}$ to temperature slightly lower than $T_{m}$ and higher than $T_{p}$ and then cooling the samples back to $293 \mathrm{~K}$. The dependences obtained for sample AT3 are presented in Fig. 6. One can see that the temperature dependences of SP energy and bandwidth at cooling are qualitatively similar to the depen- 


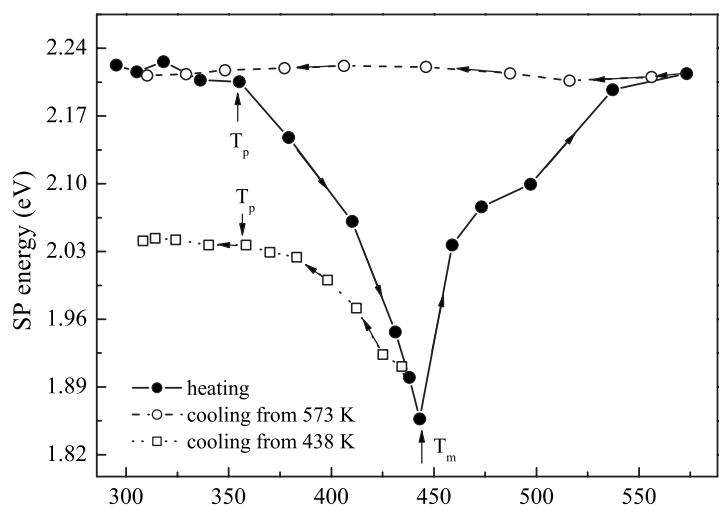

(a) Temperature (K)

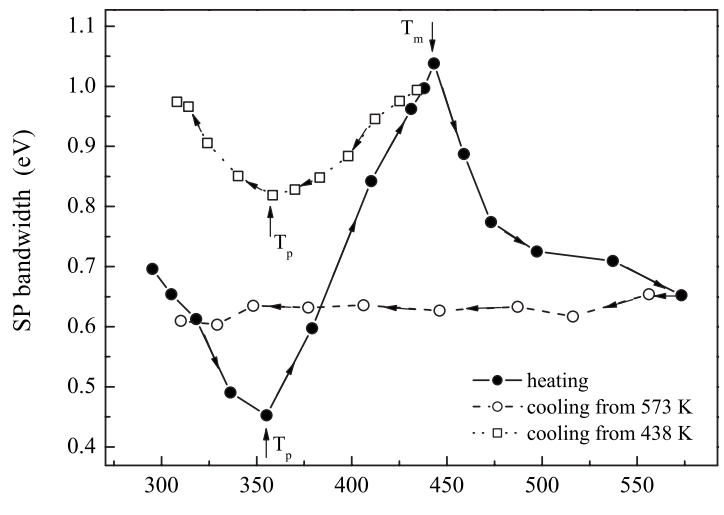

(b)

Temperature (K)

FIG. 6. Irreversibility of temperature dependence of (a) SP energy and (b) bandwidth of gold nanoparticles in sample AT3 with $f=0.34$. First experiment: heating of sample from 293 to $438 \mathrm{~K}$ exceeding the matrix softening point $T_{p}$ and lower than Au nanoparticle melting point $T_{m}$ (filled circles) and subsequent cooling to $293 \mathrm{~K}$ (open squares). Second experiment: heating of sample from 293 to $573 \mathrm{~K}$ exceeding the Au nanoparticle melting point $T_{m}$ (filled circles) and subsequent cooling to $293 \mathrm{~K}$ (open circles).

dences at heating but do not map them quantitatively. Thus, the irreversibility of temperature dependences of SP energy and bandwidth takes place. This proves our assumption of the aggregation of gold nanoparticles occurring due to softening of polymer matrix. Let us note that the softening point $T_{p}$ of PTFE matrix lowers with increase in filling factor (see Fig. 4). This reflects the well known effect of the change in mechanical, thermal, electrical, and optical properties of polymers (PTFE in part) at filling of polymer by metallic, semiconductor, or insulator particles.

At the temperature marked as $T_{m}$ the broadening and redshift of SP absorption band with increase in temperature change to sharp narrowing and blueshift. Such behavior manifests itself as strong jumplike features at $613 \mathrm{~K}$ in AT4 $(f=0.07), 553 \mathrm{~K}$ in AT2 $(f=0.20)$, and $444 \mathrm{~K}$ in AT3 $(f$ $=0.34$ ). Apparently, the processes in matrix cannot cause such drastic effects. At the temperatures close to $T_{m}$ the matrix is softened considerably, but the thermal decomposition of PTFE starts at higher temperatures. To our opinion, the following phenomena can lead to observed sharp features in temperature dependences of SP energy and bandwidth: the melting or sintering of small aggregated gold nanoparticles involving their merging and formation of larger isolated spherical nanoparticle or the solid-solid phase transition (structural transition) in nanoparticle. Later a phenomenon was observed by HRTEM technique for free gold nanoparticles $^{25}$ and by electron diffraction technique for free copper nanoparticles. ${ }^{26,27}$ It was revealed in Ref. 25 that small nanoparticles with sizes of about $5 \mathrm{~nm}$ have noncrystalline icosahedral (Ic) or decahedral (Dh) morphologies that are different from large gold nanoparticles, which have the crystalline fcc morphology. So, the structural transition like Ih $\rightarrow$ Dh can cause the observed features in temperature dependences of SP spectral characteristics. However, we have revealed in our earlier study ${ }^{19}$ that in the copper nanoparticles of the same $(5 \mathrm{~nm})$ size the above mentioned solidsolid structural transition does not manifest itself in temperature dependences of SP spectral characteristics. Since gold and copper nanoparticles have the same structure and very similar electronic properties, it is reasonable to suppose that such transition does not affect the optical properties of gold nanoparticles. It is mentioned above that a different cause of observed sharp features in temperature dependences of SP characteristics can be the merging of small aggregated nanoparticles to larger isolated ones. The transformation of the aggregate of small nanoparticles to larger isolated spherical nanoparticle would lead to considerable reduction in the interparticle interaction, which affects considerably the absorption spectra of aggregate. Correspondingly, it would be manifested in absorption spectra as blueshift and narrowing of SP band with increase in temperature. Besides this, the increase in nanoparticle size has to cause the narrowing of SP band in accordance with expression (2). Since sintering is the gradual process which occurs in quite wide temperature region, it is very unlikely that sharp features in temperature dependences of SP energy and bandwidth are caused by the sintering of aggregated gold nanoparticles. On the other hand, the blueshift of SP absorption band occurring at the increase in temperature is one of the important characteristics of the bulk melted metals. ${ }^{28}$ That effect is due to the reduction in polarizability of the melted ion core. At temperatures higher than $T_{m}$ we observed the blueshift of SP band at the increase in temperature. Therefore, it is quite reasonable to suppose that sharp jumplike features in temperature dependences of SP energy and bandwidth are the manifestation of the melting of aggregated $5 \mathrm{~nm}$ gold nanoparticles occurring at melting point marked as $T_{m}$.

We observed the similar to observed jumplike behavior of the SP energy and bandwidth earlier ${ }^{19}$ for copper nanoparticles in silica matrix. We showed there that the above features are caused by the melting of $\mathrm{Cu}$ nanoparticles. Such jumplike behavior of the thermodynamic characteristics is peculiar for the phase transition solid state-liquid state or melting. The similar jumplike dependence of heat flow on temperature in Sn nanoparticles with sizes of 26-85 $\mathrm{nm}$ was reported in Ref. 2 that was attributed to the melting of $\mathrm{Sn}$ nanoparticles. A jumplike increase in the electron-phonon scattering frequency was reported in Ref. 29 at the transition of bulk metals from the solid to liquid phase, i.e., at the bulk metal melting. Thus, the jumplike behavior of SP energy and bandwidth can be caused on the one hand by aggregated 
state of nanoparticles at their melting and on the other hand by the jumplike behavior of nanometal electronic properties occurring at the melting.

It is seen from Fig. 4 that the melting points of gold nanoparticles in studied samples are much lower than the one of bulk gold $(1337 \mathrm{~K})$ : $613 \mathrm{~K}$ for sample AT4 with $f=0.07$, $553 \mathrm{~K}$ for AT2 with $f=0.20$, and $444 \mathrm{~K}$ for AT3 with $f$ $=0.34$. The effect of melting point lowering with decrease in nanoparticle size is a well known phenomenon. Similar lowtemperature melting (at the temperatures much lower than the bulk melting point) of copper spherical nanoparticles was observed by optical spectroscopy in our recent work ${ }^{19}$ and of the copper nanorods in Ref. 30 by TEM, scanning electron microscopy, and x-ray diffraction techniques. The surrounding environment is an important factor affecting the process of nanoparticle melting. In our earlier work ${ }^{19}$ we revealed that the surrounding matrix can affect considerably the melting point of nanoparticles lowering it. Most probably, the observed quite low melting point of gold nanoparticles in studied samples is, in part, caused by interaction between the nanoparticle and surrounding matrix. Namely, the wetting of the matrix by the molten gold facilitates the melting that decreases the melting point.

Besides the effects of the melting point lowering involved by nanoparticle size and matrix effects, Fig. 4 demonstrates that (1) the melting point of Au nanoparticles decreases at the increase in filling factor and (2) the noted above jumplike features become stronger at the increase in the filling factor. This leads to suggestion that both these effects are caused by interaction between gold nanoparticles. Let us discuss these effects. The first one is the lowering of melting point of gold nanoparticles occurring at the increase in filling factor of nanoparticles in composite. To our opinion the most possible cause of this effect is the interaction between gold nanoparticles. The attractive interaction between nanoparticles (dipole-dipole interaction in case of small nanoparticles) would decrease the forces attracting the surface $\mathrm{Au}$ atoms to the volume of own nanoparticle. Therefore, the forces of interparticle interaction act as some analog of the forces acting between the atoms of liquid and atoms of solid surface in the phenomenon of wetting of solid surface by liquid. It is clear that such forces would make the atoms on the nanoparticle surface freer to detach from their positions and would lead to diffusion of surface atoms in nanoparticles. This would simplify the melting and, correspondingly, would lower the melting point of the nanoparticle. Apparently, the interparticle interaction would be sufficiently stronger between the aggregated gold nanoparticles than between isolated ones. So, the aggregation would cause the considerable lowering of the nanoparticle melting point. It is clear that the higher the filling factor is, the larger and denser the aggregate is. So, the melting point has to lower with increase in filling factor. The interparticle interaction would affect the interfacial energies of solid, liquid, and vapor phases of gold. The melting point of nanoparticle depends on such energies. ${ }^{7}$ Consequently, the interparticle interaction would affect the melting point of nanoparticle. Let us note that the proposed explanation of the influence of interparticle interaction on the melting point of nanoparticle is only our assumption. Therefore, the problem of the mechanism of influence of the interaction on melting point of metal nanoparticles is still the open problem that needs a detailed theoretical study.

The second observed effect is that the observed jumplike features in temperature dependences of SP spectral characteristics become stronger at the increase in filling factor. It was recently observed ${ }^{19,31}$ that the temperature dependences of SP absorption band for small copper and gold nanoparticles are quite different from the ones for large particles. Namely, for small particles the behavior of SP energy and bandwidth with temperature is monotonic and is not characterized by abrupt jump at melting point. Let us note that temperatures reached in experiments ${ }^{19,31}$ with small $\mathrm{Cu}$ and Au nanoparticles are sufficient for melting of nanoparticles. So, the melting of small nanoparticles occurs in other way than for large ones. To our opinion, there exist two possible causes of effect of strengthening of jumplike features in temperature dependences of SP energy and bandwidth at increase in filling factor. The first one is discussed above. It consists of aggregation of small solid nanoparticles due to softening of matrix with consequent melting of the aggregated nanoparticles. Such effect would lead to jumplike change in broadening and redshift of SP band with increase in temperature to its narrowing and blueshift occurring at $T_{m}$. It is apparent that the higher the filling factor, the stronger the aggregation and, correspondingly, the stronger jump has to be observed. Let us note that jumplike features have to be presented in temperature dependences of SP energy and bandwidth even if melting of small $5 \mathrm{~nm}$ nanoparticles occurs gradually, i.e., without jump in their thermodynamic characteristics. So, only the melting of small isolated nanoparticles would not be manifested in temperature dependences of SP spectral characteristics. The second possible cause of the strengthening of jumplike manifestation of melting of gold nanoparticles with increase in filling factor is following one. The molecular dynamics simulations of the solid-liquid phase transitions in various metal nanoparticles were performed in Refs. 7 and 11-13. It was shown in Ref. 11 that some characteristic particle size (designated as $d_{2}$ ) exists. Likely to melting point, $d_{2}$ depends on the interfacial energies of solid, liquid, and vapor phases of metal. For particles with size larger than $d_{2}$ the solid-liquid phase coexistence is stable. The melting of such particles is first-order transition, i.e., the melting occurs likely to bulk metals. For particles smaller than $d_{2}$ the solid-liquid coexistence is unstable or metastable. The melting of such nanoparticles occurs gradually, i.e., without the jumplike changes in thermodynamic parameters. It is seen in Fig. 4 that in sample AT4 with lowest filling factor of 0.07 the jumplike features in temperature dependences of SP energy and bandwidth are manifested quite slightly, i.e., the melting transition occurs most gradually. That approaches such melting to the case of isolated small copper ${ }^{19}$ and gold ${ }^{31}$ nanoparticles with sizes smaller than $d_{2}$ which melt gradually, i.e., without the nonmonotonic jumplike features in temperature dependences of SP spectral characteristics. So, the size $(5 \mathrm{~nm})$ of gold nanoparticles in a sample with $f=0.07$ is close (a little higher) to $d_{2}$. Meanwhile, at the increase in filling factor the jumplike 
features become stronger (Fig. 4). Therefore, one can suppose that the value of critical size $d_{2}$ depends on the filling factor, namely, $d_{2}$ decreases with increase in $f$, i.e., with strengthening of interaction between the gold nanoparticles. The origin of dependence $d_{2}(f)$ is similar to the dependence of melting point on filling factor $\left[T_{m}(f)\right]$ discussed above. Namely, the interparticle interaction would affect the interfacial energies of solid, liquid, vapor phases of gold, and, correspondingly, the characteristic size $d_{2}$. So, at the increase in $f$ the gold nanoparticle size $(5 \mathrm{~nm})$ exceeds the critical size $d_{2}$ more and more. Correspondingly, the melting of gold nanoparticles manifests itself in temperature dependences of SP energy and bandwidth as more and more strong jumplike features. Let us note that the proposed mechanism of dependences $T_{m}(f)$ and $d_{2}(f)$ is our assumption that needs a detailed theoretical study.

It is seen from Fig. 4(b) that the SP bandwidth decreases with increase in temperature in temperature region from $T_{m}$ to about $800 \mathrm{~K}$. The SP energy in this temperature region increases with increasing temperature. As we note above it is the result of melting of small $(5 \mathrm{~nm})$ aggregated nanoparticles and their subsequent merging with the formation of larger spherical nanoparticles. Since the size of nanoparticle formed at the merging of several small melted nanoparticles is larger than these small ones, the melting point of large gold nanoparticles is higher than for small ones. Considering the strong size dependence of melting point of nanoparticles in nanometer-sized region where gold nanoparticles in nonannealed samples are, the melting point of large nanoparticles has to be sufficiently higher than for small ones. So, just after its formation due to merging, the large nanoparticles would be supercooled and after lapse of time of about $1 \mathrm{~ns}^{7}$ it would freeze (crystallize). In other words, the merging of small spherical liquid nanoparticles would lead to the formation of large spherical solid nanoparticles. It has to be noted that the formation of large solid nanoparticles can occur as well due to the merging of already solid nanoparticle and the liquid one. To check the assumption of the formation of large solid spherical gold nanoparticles we measured the temperature dependences of SP energy and bandwidth at heating of samples to temperatures exceeding considerably the melting point of nanoparticles (Fig. 6, filled circles) and at consequent cooling of samples to room temperatures (Fig. 6 , open circles). It is clearly seen that dependences are fully irreversible. The discussion outlined above explains the fact why at cooling of the samples down to room temperatures any remarkable features in the temperature dependences of SP spectral characteristics similar to jumplike ones observed at the heating were not observed, i.e., the obtained dependences are fully irreversible. In fact, at cooling the composite consists of large solid gold nanoparticles with melting point higher than temperatures reachable in our experiments. Consequently, at cooling the temperature dependences of SP energy and bandwidth for large solid particles do not have the jumplike features. Since the merging of nanoparticles and the corresponding freezing of large gold nanoparticles occur in wide temperature and nanoparticle size range (at the tem-

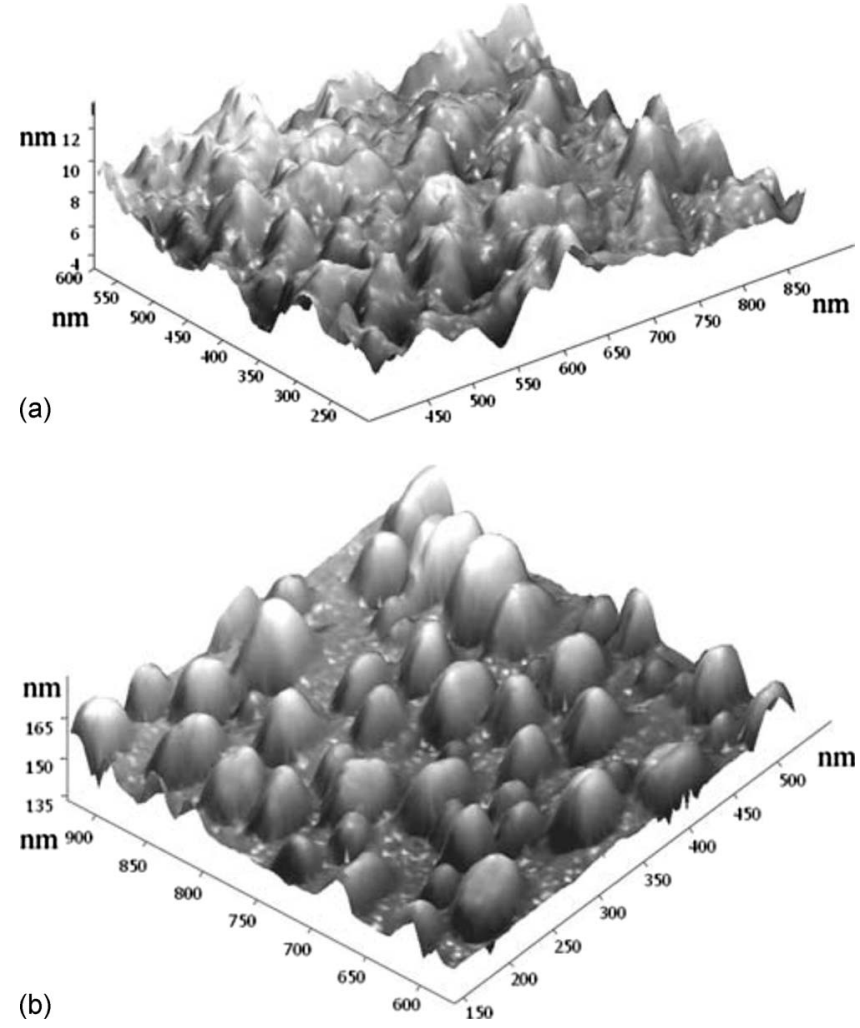

FIG. 7. AFM images of Au/PTFE nanocomposite with $f=0.34$ (a) before and (b) after heating up to $936 \mathrm{~K}$.

peratures higher than $T_{m}$ and sizes larger than $5 \mathrm{~nm}$ ) the freezing does not manifest itself in the temperature dependences of SP spectral characteristics.

Let us note the redshift of SP absorption band at the increase in temperature for all samples observed at temperatures higher than about $800 \mathrm{~K}$ (Fig. 4). The decrease in SP energy reflects most probably the fact of increase in effective dielectric constant of medium surrounding the gold nanoparticles, as the SP frequency $\omega_{\text {sp }}$ depends on the dielectric constant of matrix $\epsilon_{m}$ as $\omega_{\mathrm{sp}}=\omega_{p} / \sqrt{1+2 \epsilon_{m}}$. At these high temperatures the process of thermal decomposition of PTFE occurs. Since the nanocomposite films are quite thin (100$300 \mathrm{~nm}$ ), the decomposition of PTFE matrix would lead to the thinning of the film and to the precipitation of the gold nanoparticles onto glass substrate. Since the glass has higher dielectric constant (2.10) than PTFE (1.77), the SP energy in gold nanoparticles precipitated onto glass has to be lower than in nanoparticles in PTFE matrix. We performed the AFM study of initial thermally nontreated Au/PTFE nanocomposite samples and the ones heated up to $936 \mathrm{~K}$. The obtained AFM images for sample AT3 with a filling factor of 0.34 are shown in Fig. 7. It is seen that the image of thermally treated sample [Fig. 7(b)] differs considerably from the image of nontreated one [Fig. 7(a)]. The surface of nontreated sample has chaotic relief with mean height of hills of about $3 \mathrm{~nm}$. The surface of thermally treated sample is sufficiently smoother. It is clearly seen the gold nanoparticles with mean vertical size of about $25 \mathrm{~nm}$ on this surface. So, the AFM image proves the fact of the precipitation of gold nanoparticles onto glass substrate due to the thermal decomposition of polymer PTFE matrix. Since the size of gold 
nanoparticles in a thermally treated sample taken from AFM image [Fig. 7(b)] is significantly larger (about $25 \mathrm{~nm}$ ) than in nontreated one, the AFM study proves the fact of increase in size of gold nanoparticle at the heating of Au/PTFE nanocomposite samples. Consequently, the increase in size of nanoparticles proves the fact of merging of gold nanoparticles and, consequently, the fact of their melting.

\section{CONCLUSIONS}

In conclusion, we studied the temperature behavior of the absorption spectra of Au/PTFE nanocomposites with various filling factors in temperature range of 77-936 K. The obtained temperature dependences of SP energy and bandwidth in gold nanoparticles with size of $5 \mathrm{~nm}$ prove the aggregation of nanoparticles due to thermal softening of polymer matrix and consequent melting of nanoparticles with formation of isolated large (about $25 \mathrm{~nm}$ ) nanoparticles. The melting of gold nanoparticles occurs at temperatures considerably lower than the melting point of bulk gold. The interaction between the gold nanoparticles significantly affects the melting of nanoparticles. When the filling factor increases the melting point of gold nanoparticles decreases and the spectral signature of their melting becomes stronger.

\section{ACKNOWLEDGMENTS}

The authors thank Dr. Yu. Kolomzarov for film deposition, Dr. S. Kondratenko for AFM measurements, and Dr. A. Pinchuk for helpful discussions and careful reading of the manuscript. Research was partially made in the frame of STCU (Grant No. 3480).

${ }^{1}$ M. Dippel, A. Maier, V. Gimple, H. Wider, W. E. Evenson, R. L. Rasera, and G. Schatz, Phys. Rev. Lett. 87, 095505 (2001).

${ }^{2}$ H. Jiang, K. Moon, H. Dong, F. Hua, and C. P. Wong, Chem. Phys. Lett. 429, 492 (2006).

${ }^{3}$ Z. L. Wang, J. M. Petroski, T. C. Green, and M. A. El-Sayed, J. Phys. Chem. B 102, 6145 (1998).

${ }^{4}$ M. Alcoutlabi and G. B. McKenna, J. Phys.: Condens. Matter 17, R461
(2005).

${ }^{5}$ F. Ercolessi, W. Andreoni, and E. Tosatti, Phys. Rev. Lett. 66, 911 (1991).

${ }^{6}$ R. N. Barnett and U. Landman, Phys. Rev. B 44, 3226 (1991).

${ }^{7}$ L. J. Lewis, P. Jensen, and J.-L. Barrat, Phys. Rev. B 56, 2248 (1997).

${ }^{8}$ J. Wang, X. Chen, G. Wang, B. Wang, W. Lu, and J. Zhao, Phys. Rev. B 66, 085408 (2002).

${ }^{9}$ S. Link, Z. L. Wang, and M. A. El-Sayed, J. Phys. Chem. B 104, 7867 (2000).

${ }^{10}$ P. Buffat and J.-P. Borel, Phys. Rev. A 13, 2287 (1976).

${ }^{11}$ D. Schebarchov and S. C. Hendy, J. Chem. Phys. 123, 104701 (2005).

${ }^{12}$ D. Schebarchov and S. C. Hendy, Phys. Rev. Lett. 96, 256101 (2006).

${ }^{13}$ C. L. Cleveland, W. D. Luedtke, and U. Landman, Phys. Rev. B 60, 5065 (1999).

${ }^{14}$ T. Bachels, H.-J. Güntherodt, and R. D. Schåfer, Phys. Rev. Lett. 85, 1250 (2000).

${ }^{15}$ T. Castro, R. Reifenberger, E. Choi, and R. P. Andres, Surf. Sci. 234, 43 (1990).

${ }^{16}$ T. P. Martin, U. Naher, H. Schaber, and U. Zimmermann, J. Chem. Phys. 100, 2322 (1994).

${ }^{17}$ M. Schmidt, R. Kusche, W. Kronmuller, B. von Issendorff, and H. Haberland, Phys. Rev. Lett. 79, 99 (1997).

${ }^{18}$ L. Gråbæk, J. Bohr, H. H. Andersen, A. Johansen, E. Johnson, L. SarholtKristensen, and I. K. Robinson, Phys. Rev. B 45, 2628 (1992).

${ }^{19}$ O. A. Yeshchenko, I. M. Dmitruk, A. A. Alexeenko, and A. M. Dmytruk, Phys. Rev. B 75, 085434 (2007).

${ }^{20}$ K. P. Gritsenko and A. M. Krasovsky, Chem. Rev. (Washington, D.C.) 103, 3607 (2003).

${ }^{21}$ K. P. Grytsenko, D. O. Grynko, M. V. Sopinskyy, and S. Schrader, Data Record. Storage Process. 4, 3 (2004).

${ }^{22}$ B. D. Cullity and S. R. Stock, Elements of X-Ray Diffraction, 3rd ed. (Prentice-Hall, Englewood Cliffs, NJ, 2001).

${ }^{23}$ M. G. Bawendi, A. R. Kortan, M. L. Steigerwald, and L. E. Brus, J. Chem. Phys. 91, 7282 (1989).

${ }^{24}$ U. Kreibig and M. Vollmer, Optical Properties of Metal Clusters (Springer, New York, 1995).

${ }^{25}$ K. Koga, T. Ikeshoji, and K. I. Sugawara, Phys. Rev. Lett. 92, 115507 (2004).

${ }^{26}$ D. Reinhard, B. D. Hall, P. Berthoud, S. Valkealahti, and R. Monot, Phys. Rev. Lett. 79, 1459 (1997).

${ }^{27}$ D. Reinhard, B. D. Hall, P. Berthoud, S. Valkealahti, and R. Monot, Phys. Rev. B 58, 4917 (1998).

${ }^{28}$ J. C. Miller, Philos. Mag. 20, 1115 (1969).

${ }^{29}$ T. E. Faber, in The Physics of Metals, edited by J. M. Ziman (Cambridge University Press, Cambridge, England, 1969), Chap. 6, p. 282.

${ }^{30}$ T. Karabacak, J. S. DeLuca, and P.-I. Wang, J. Appl. Phys. 99, 064304 (2006).

${ }^{31}$ D. Dalacu and L. Martinu, Appl. Phys. Lett. 77, 4283 (2000). 